\title{
INTERVENCIÓN EDUCATIVA PARA MEJORAR LA CULTURA AMBIENTAL Y FITOTERAPÉUTICA EN ESTUDIANTES DEL NIVEL SECUNDARIO DE UNA INSTITUCIÓN EDUCATIVA DE LA PROVINCIA DE JULCÁN, LA LiBERTAD
}

\section{EDUCATIONAL INTERVENTION TO IMPROVE CULTURE ENVIRONMENTAL AND PHYTOTHERAPEUTIC IN THE STUDENTS OF THE SECONDARY LEVEL of educATive institution of JulCAN'S PROVINCE, La Libertad}

\author{
Ericson Félix Castillo Saavedra* \\ Segundo Félix Castillo Viera** \\ Paquita Micaela Carranza López***
}

\begin{abstract}
RESUMEN
I a presente investigación de tipo cuantitativa, explicativa y evaluativa, con diseño cuaLsiexperimental tuvo como propósito mejorar la cultura ambiental y fitoterapéutica de los estudiantes del nivel secundario de una institución educativa de la provincia de Julcán, departamento La Libertad, a través de la planificación, organización, ejecución y evaluación de una intervención educativa. La cultura ambiental y fitoterapéutica fue medida mediante un test de 54 ítems que abarcó las dimensiones de calidad ambiental y calidad de vida, categorizados por medio de una escala de Likert. La muestra fue estimada mediante técnica no probabilística intencional que reflejó la información existente de la población de estudiantes del nivel secundario. Los resultados encontrados en el pretest (135.6 puntos) y postest (225.3 puntos) del grupo experimental, y en comparación con el grupo control (137.3 puntos) evidenciarían que la intervención educativa mejoró significativamente $(\mathrm{p}<0.01)$ la cultura ambiental y fitoterapéutica de los estudiantes del nivel secundario de una institución educativa.
\end{abstract}

Palabras clave: Intervención educativa, cultura, fitoterapia, medio ambiente

* Doctor en Ciencias Biomédicas. Químico Farmacéutico. Docente de la Escuela de Farmacia y Bioquímica. Universidad Católica Los Ángeles de Chimbote. Universidad Nacional de Trujillo. ericson_fcs@ hotmail.com

** Doctor en Ciencias Biológicas. Biólogo. Universidad Nacional de Trujillo.

*** Licenciada en Educación. Universidad Nacional de Trujillo. Trujillo. 


\section{ABSTRACT}

The present research of type quantitative, explanatory and evaluative, with quasiexperimental design had as propose improve the environmental and phytotherapeutic culture of the students of the secondary level of the educative institution of Julcan's district, department The Libertad, for which realized the planning, organization, execution and evaluation of an educational intervention. The environmental and phytotherapeutic culture was measured by a test of 54 questions, which it had as dimensions environmental quality and quality of life, categorized by a Likert scale. The sample was estimated by intentional non-probabilistic technique that reflected the existing information of the population of secondary students. The results found in the pretest (135.6 points) and postest (225.3 points) of the experimental group, and in comparison with the group control (137.3 points) they would demonstrate that the educational intervention improved significantly $(\mathrm{p}<0.01)$ the environmental and phytotherapeutic culture of the students of the secondary level of the Javier Heraud school.

Keywords: Educational intervention, culture, phytotherapy, environment.

\section{INTRODUCCIÓN}

A nivel mundial, las experiencias educativas tradicionales y sus problemas metodológicos específicos, como la carencia de una visión global en los programas educativos, la tendencia a acentuar la especialización y a fomentar una percepción estrecha de la realidad, que no suscitan una respuesta activa de los alumnos, obstaculizaría el desarrollo de la Educación Ambiental (EA) (1).

La cultura ambiental es la educación que el ser humano presenta sobre el cuidado y preservación del medio ambiente, y se encuentra relacionada con la educación ambiental (EA) $(1,2,3)$.

En el contexto actual, el desarrollo de una cultura ambiental supone un cambio de concepción del hombre sobre sí mismo y sobre su lugar en el mundo, y consecuentemente de su lugar respecto con los otros hombres, con la sociedad y con la naturaleza (2).

La cultura ambiental y fitoterapéutica en la presente propuesta está relacionada con los conocimientos sobre el cuidado y preservación de los recursos naturales, y la utilización de la fitoterapia como medio alternativo terapéutico para el tratamiento de patologías (4).

El conocimiento sobre el cuidado del medio ambiente involucra que el ser humano conozca como clasificar la basura separando la orgánica de la inorgánica, para que pueda ser reciclada. Entre los materiales reciclables se encuentran: el papel, el vidrio, el plástico y el metal, los cuales, bien limpios, pueden volverse a usar dentro de la casa, o bien, venderse a centros que se dedican a recopilarlos $(3,4)$. 
La fitoterapia constituye una herramienta terapéutica dentro de todo el abanico de posibilidades que nos brinda la terapéutica actual, y el conocimiento de su utilización para tratar algunas patologías es realmente útil, siendo necesario hacer un uso racional basado en un uso apropiado de los preparados a base de plantas medicinales (5 - 9).

La EA es un proceso dinámico y participativo, que busca despertar en la población una conciencia que le permita identificarse con la problemática ambiental tanto a nivel general (mundial), como a nivel específico (medio donde vive); busca identificar las relaciones de interacción e independencia que se dan entre el entorno (medio ambiente) y el hombre, así como también se preocupa por promover una relación armónica entre el medio natural y las actividades antropogénicas a través del desarrollo sostenible $(2,10)$.

El conocimiento ambiental es un proceso complejo, que incluye la obtención, análisis y sistematización por parte del individuo de la información proveniente de su entorno, social por naturaleza, este constituye un paso importante para su comprensión a través de acciones concretas, que a su vez, influyen en el desarrollo de estos conocimientos (11)

Los fundamentos teóricos y metodológicos se sustentan en la concepción filosófica dialéctica - materialista y se justifica por las necesidades sociales relevantes, actuales y perspectivas, de una formación ambiental que le proporciona al hombre el marco teórico integrador para la orientación en el complejo sistema de interacciones cognitivas, económicas, políticas e ideológicas (12).

En este sentido, y en base a lo anteriormente mencionado, las actividades programadas en la intervención educativa favorecieron el desarrollo de una metodología activa que estuvo basada en dos principios didácticos fundamentales: el aprendizaje constructivo y el aprendizaje significativo. De esta manera los alumnos estuvieron capacitados para comprender las relaciones con el medio al cual pertenecen y dar respuestas de forma activa, participativa y reflexiva a los problemas de su ámbito más próximo.

La formación a nivel secundario en las instituciones educativas de la provincia de Julcán adolece de las mismas limitaciones de la educación básica primaria, aunque, en este caso, el interés en el tema del profesor de Ciencia, tecnología y Ambiente (CTA) puede llegar a desempeñar un papel importante, mediante charlas o ejemplos que puede sacar de su propia experiencia $(13,14)$.

Los principales problemas ambientales de la provincia de Julcán están relacionados a la pérdida de la regulación hídrica y calidad del agua, pérdida y deterioro de la biodiversidad animal y vegetal, pérdida del bosque y degradación genética de las especies, por el aprovechamiento irracional de las mismas, bajo grado de conciencia para comprender, cumplir o hacer cumplir las normativas ambientales por parte de las autoridades y la comunidad en general y poca conciencia de la población para promover el desarrollo sostenible en las diferentes practicas productivas que se realizan.

Con base en lo mencionado, la finalidad del estudio fue mejorar la cultura ambiental y fitoterapéutica de los estudiantes del nivel secundario de una institución educativa de la provincia de Julcán. 


\section{MATERIAL Y MÉTODOS}

El tipo de investigación fue cuantitativo, explicativo y evaluativo, en el que se manipuló intencionalmente una variable independiente (supuestas causas-antecedentes: intervención educativa), para analizar las consecuencias que la manipulación tiene sobre una o más variables dependientes (supuestos efectos-consecuentes: cultura ambiental y fitoterapéutica), dentro de una situación de control para el investigador.

Del mismo modo, se utilizó el diseño cuasi-experimental, donde los sujetos no se asignaron al azar a los grupos, ni se emparejaron, sino que dichos grupos ya estuvieron formados antes del experimento.

La población representó a la muestra y fue estimada mediante técnica muestral no probabilística intencional en forma tal que reflejó la información existente de la población de estudiantes del nivel secundario de la institución educativa (3).

La variable independiente intervención educativa abarcó las siguientes dimensiones: planificación, organización, ejecución y evaluación, se utilizó como técnica la observación, y como instrumento de recolección de datos la lista de cotejo.

La variable dependiente cultura ambiental y fitoterapéutica abarcó las siguientes dimensiones: calidad ambiental y calidad de vida, se utilizó como técnica la encuesta, y como instrumento de recolección de datos el pretest y postest.

La t student comparativa con una significancia del 1\% permitió evaluar el nivel de validez de los resultados y el nivel de significancia de las diferencias antes y después entre las variables estudiadas mediante el método inductivo-deductivo (3).

\section{RESULTADOS}

Tabla 1. Nivel de cultura ambiental y fitoterapéutica en el grupo control antes de la intervención educativa en estudiantes del nivel secundario de una institución educativa de la provincia de Julcán, La Libertad

\begin{tabular}{cccccc}
\hline & \multicolumn{3}{c}{ Cultura ambiental y fitoterapéutica } & \multirow{2}{*}{ Significancia } \\
\cline { 2 - 4 } Nivel & \multicolumn{2}{c}{ Calidad ambiental } & \multicolumn{2}{c}{ Calidad de vida } & \multirow{2}{*}{$\mathrm{X}^{2}$} \\
\cline { 2 - 5 } & $\mathrm{n}$ & $\%$ & $\mathrm{~N}$ & $\%$ & \\
\hline Bajo & 24 & 75 & 20 & 62,5 & \\
Medio & 8 & 25 & 12 & 37,5 & \multirow{2}{*}{$\mathrm{p}<0.01$} \\
Alto & 0 & 0 & 0 & 0 & \\
\hline
\end{tabular}


Tabla 2. Nivel de cultura ambiental y fitoterapéutica en el grupo control después de la intervención educativa en estudiantes del nivel secundario de una institución educativa de la provincia de Julcán, La Libertad.

\begin{tabular}{rcccccc}
\hline \multirow{2}{*}{ Nivel } & \multicolumn{3}{c}{ Cultura ambiental y fitoterapéutica } & \multirow{2}{*}{ Significancia } \\
\cline { 2 - 4 } & \multicolumn{2}{c}{ Calidad ambiental } & \multicolumn{2}{c}{ Calidad de vida } & \multirow{2}{*}{$\mathrm{X}^{2}$} \\
\cline { 2 - 4 } & $\mathrm{n}$ & $\%$ & $\mathrm{n}$ & $\%$ & \\
Bajo & 22 & 68,8 & 20 & 62,5 & \\
Medio & 10 & 31,2 & 12 & 37,5 & $\mathrm{p}<0.01$ \\
Alto & 0 & 0 & 0 & 0 & \\
\hline
\end{tabular}

Tabla 3. Nivel de cultura ambiental y fitoterapéutica en el grupo experimental antes de la intervención educativa en estudiantes del nivel secundario de una institución educativa de la provincia de Julcán, La Libertad.

\begin{tabular}{cccccc}
\hline \multirow{2}{*}{ Nivel } & \multicolumn{3}{c}{ Cultura ambiental y fitoterapéutica } & \multirow{2}{*}{ Significancia } \\
\cline { 2 - 4 } & \multicolumn{2}{c}{ Calidad ambiental } & \multicolumn{2}{c}{ Calidad de vida } & \multirow{2}{*}{$\mathrm{X}^{2}$} \\
\cline { 2 - 4 } & $\mathrm{n}$ & $\%$ & $\mathrm{n}$ & $\%$ & \\
Bajo & 23 & 71,9 & 23 & 71,9 & \\
Medio & 9 & 28,1 & 9 & 28,1 & $\mathrm{p}<0.01$ \\
Alto & 0 & 0 & 0 & 0 & \\
\hline
\end{tabular}

Tabla 4. Nivel de cultura ambiental y fitoterapéutica en el grupo experimental después de la intervención educativa en estudiantes del nivel secundario de una institución educativa de la provincia de Julcán, La Libertad.

\begin{tabular}{cccccc}
\hline \multirow{2}{*}{ Nivel } & \multicolumn{4}{c}{ Cultura ambiental y fitoterapéutica } & \multirow{2}{*}{ Significancia } \\
\cline { 2 - 4 } & \multicolumn{2}{c}{ Calidad ambiental } & \multicolumn{2}{c}{ Calidad de vida } & \multirow{2}{*}{$\mathrm{X}^{2}$} \\
\cline { 2 - 4 } Bajo & $\mathrm{n}$ & $\%$ & 0 & 0 & \\
Medio & 10 & 31,2 & 9 & 28,1 & $\mathrm{p}<0.01$ \\
Alto & 22 & 68,8 & 23 & 71,9 & \\
\hline
\end{tabular}

Tabla 5. Estadísticos descriptivos de la cultura ambiental y fitoterapéutica antes y después de la intervención educativa en el grupo control.

\begin{tabular}{ccccc}
\hline $\begin{array}{c}\text { Cultura ambiental y } \\
\text { fitoterapéutica }\end{array}$ & Media & N & Prueba Z & Significancia \\
\hline Antes & 68,0 & 32 & 1,98 & $\mathrm{p}>0.05$ \\
Después & 70,3 & 32 & & N.S. \\
\hline
\end{tabular}

Tabla 6. Estadísticos descriptivos de la cultura ambiental y fitoterapéutica antes y después de la intervención educativa en el grupo experimental.

\begin{tabular}{ccccc}
\hline $\begin{array}{c}\text { Cultura ambiental y fitote- } \\
\text { rapéutica }\end{array}$ & Media & N & Prueba Z & Significancia \\
\hline Antes & 68,2 & 32 & 97,6 & $\mathrm{p}<0.01$ \\
Después & 117,0 & 32 & & \\
\hline
\end{tabular}




\section{DISCUSIÓN}

En las tablas 1 y 2 se observa en el grupo control que la cultura ambiental y fitoterapéutica expresada como calidad ambiental y calidad de vida reportó $75 \%$ y $62.5 \%$ en el pretest, y $68.8 \%$ y $62.5 \%$ en el postest respectivamente, alcanzando un nivel bajo, y demostraría según indican Gonzales (15), Súcar (16) y Terrón (17) la carencia de conocimientos sobre temáticas relacionadas a la preservación y protección del medio ambiente, lo cual pone de manifiesto que las interacciones entre el ser humano y la naturaleza son tan complejas y pluridimensionales, que la meta de la comprensión y el mejoramiento de esta relación, requiere del apoyo de casi todas las disciplinas y cualquier acción encaminada a resolver un problema importante del medio ambiente, dependería de la participación activa de un amplio conjunto de personas e instituciones.

Los resultados en el nivel bajo para las dimensiones calidad ambiental y calidad de vida son semejantes a lo encontrado por Nodarse (18), quien revela que los estudiantes en esta etapa de sus vidas aún no han logrado apropiarse en profundidad de la dimensión que caracteriza al medio ambiente, todavía no presentan el sentido de pertenecer a ese medio ambiente que les pertenece, espacio donde las relaciones socioculturales y políticas ocupan un papel determinante.

En base a lo observado en las tablas 01 y 02, Molina (19), Fernández y Fallas (20) y Carrero y García (21) reiteran la necesidad de trabajar en la consolidación de una conciencia ambiental a través de programas basados en educación ambiental, consistentes en conocer valores y aclarar conceptos con el objeto de fomentar aptitudes y actitudes necesarias para comprender y apreciar las interrelaciones entre el hombre, su cultura y su medio biofísico; donde el individuo pueda advertir su incidencia negativa sobre todo lo que lo rodea.

De la misma forma, en las tablas 3 y 4 se hace referencia a la cultura ambiental y fitoterapéutica en el grupo experimental expresada como calidad ambiental y calidad de vida, encontrando $71.9 \%$ y $71.9 \%$ en el nivel bajo para el pretest, y $68.8 \%$ y $71.9 \%$ en el nivel alto para el postest respectivamente; evidenciando, que la adquisición de conocimientos y valores en educación ambiental podría provocar cambios actitudinales y comportamentales, mediante métodos pedagógicos constructivistas basados en el aprendizaje significativo (22).

El cambio significativo en el postest hacia el nivel alto (tabla 4) se basó en evitar conocimientos no comprensibles, es decir, en intentar que el educando descubra un significado a los conceptos ambientales y fitoterapéuticos que aprende, de manera que se puedan relacionar adecuada y coherentemente con los conceptos ya aprendidos con anterioridad, presentes en su estructura cognitiva. El aprendizaje significativo es opuesto al aprendizaje sólo memorístico, en el que el educando puede no dar significado a lo que aprende (23).

Así también, el abordaje de la fitoterapia evidenció claramente la mejor relación entre una situación de vida con calidad humana que promovió tranquilidad, paz, alegría y confianza, y la presencia de un ambiente con plantas en la institución educativa motivó al estudiante a la adquisición de nuevos conocimientos, frente a la relación en un am- 
biente en el que no hay plantas. En este sentido, se puede inferir que existe una preferencia por los espacios con plantas entre los estudiantes de la institución educativa. La presencia de plantas influye permanentemente en el estado de ánimo y en la percepción vital, aunque las diferencias no son significativas sí son persistentes (24).

$\mathrm{Al}$ respecto, Gonzales (24) y Zeballos (25) sostienen que los alumnos que viven su experiencia de estudio en una institución educativa cuyos ambientes poseen plantas tienen mayor disposición a una mejor calidad de vida que los que no lo tienen y además experimentarían una mayor inquietud por el cuidado del ambiente y valorarían mejor su presencia.

De este modo, la diferencia significativa reflejada en el postest respecto al pretest se debió a que la intervención educativa permitió a los estudiantes desarrollar la capacidad de diálogo como modo de aclarar sus propias opiniones, respetar las ajenas, explorar diferentes puntos de vista, siendo fuente inagotable de conocimientos, además, permitió sentar las bases para el desarrollo de actividades concretas hacia el ambiente, partiendo de que el trabajo realizado debe estar organizado para motivar el análisis, el cuestionamiento, la indagación fenomenológica, la interpretación y evaluación de información ambiental, mediante la experiencia directa $(26,27)$.

La enseñanza de las ciencias naturales en la Educación Básica Regular busca el desarrollo de un pensamiento crítico y reflexivo acerca del ambiente por los estudiantes; sin embargo, las estrategias didácticas utilizadas en la actualidad se centran en la memorización y repetición de conocimientos, sin promover la utilización de los espacios en los que se desarrolla el estudiante.

Vidal, Pellegrini y Raimúndez (28) refieren en un estudio realizado que la implementación de un sendero de interpretación botánico en un bosque, constituye un medio didáctico que garantiza experiencias activas que vinculan al estudiante con el ambiente tanto en lo sensorial como en lo cognitivo. Del mismo modo, Bernal y Meléndez (29) señalan que el ecoturismo y los principales problemas detectados por la comunidad como la contaminación y la tala de vegetación representarían herramientas importantes como estrategias para llevar a cabo programas de educación ambiental en niños y adultos.

La intervención educativa destaca la importancia del papel activo del aprendiz en su interrelación con el medio ambiente y la fitoterapia, responsabilizándole en su propio proceso pedagógico. Por lo expuesto, se vislumbra, entonces, la necesidad de utilizar recursos didácticos en una combinación que permita la integración de las experiencias de enseñanza y aprendizaje, enmarcadas dentro de los lineamientos de la Educación Ambiental, como mecanismo de mejorar el proceso formativo de los estudiantes de la institución educativa.

Del mismo modo, en la tablas 5 se observó que no existe diferencia en el grupo control, mientras que en la tabla 6 se evidencia diferencia significativa $(\mathrm{p}<0.01)$ respecto a la cultura ambiental y fitoterapéutica, lo cual indica que la intervención educativa permitió cambiar el nivel cultural, y posteriormente la percepción de los estudiantes respecto a su entorno, y estaría corroborando que en el proceso de ejecución se ejerció en general 
una influencia positiva sobre los alumnos, lo que lleva a establecer que la estructura del programa permitió tomar en cuenta la problemática ambiental de su entorno, explicando sus causas y consecuencias pero sin perder su interdependencia con la problemática ambiental global; tal como lo sugiere Novo (30) y Orejuela (3), quienes enfatizan que la educación ambiental debe ser contextual, sin descuidar el material humano al que se quiere llegar.

El cambio cognoscitivo reflejado en la tabla 6, se relaciona con lo establecido por Andraca y Sampedro (31), quien afirma que la educación ambiental parte de que si cada persona es consciente de su responsabilidad, conoce su comportamiento y es alcanzada suficientemente en su sensibilidad, puede iniciarse un cambio social en el sentido de la racionalidad ecológica y la equidad entre los pueblos; y puesto en práctica en la ejecución de la intervención educativa.

Respecto a la fitoterapia y su evaluación en el pretest y postest, el desconocimiento inicial de los estudiantes acerca de los fitofármacos, se transformó en conocimiento alto después de la intervención, y coincidiría con lo encontrado por Ismael (32), que su estudio realizado contribuyo a elevar los conocimientos sobre las plantas medicinales, lo cual facilitó la introducción de temáticas relacionadas al uso adecuado de la fitoterapia en los tratamientos de diferentes afecciones.

La intervención educativa respecto a la fitoterapia permitió a los estudiantes conocer la medicina tradicional y natural, no como un método alternativo motivado por cuestiones de índole económica como solución a los problemas de desabastecimiento de medicamentos que existen en los puestos de salud, sino como refiere Mostacero (33), una verdadera disciplina científica que es necesario estudiar, perfeccionar y desarrollar permanentemente, por sus demostradas ventajas científicas.

La medicina tradicional y natural forma parte importante del acervo cultural de la humanidad, y en Julcán se encontró una gran variedad de especies vegetales, que inicialmente desconocían su utilidad, pero que gracias a la intervención educativa, se inició un proceso de capacitación, que no solo incluía a los estudiantes de la institución educativa, sino también a los padres de familia que se sintieron motivados a utilizar esta disciplina poco difundida.

Los estudiantes en el postest presentaron un cambio de actitud positiva hacia la fitoterapia, tal como sugiere Rengifo, Flores y Guevara (34), que a partir de la construcción de un proceso de concientización sobre la utilización de las plantas medicinales, insertándose como mecanismo potenciador en sus procesos de formación, debido a que consideraron que cada país y región del mundo presenta características propias, en franca dependencia de los recursos disponibles en ellos, tomando como base además la idiosincrasia de sus habitantes.

La fitoterapia promovió el aprendizaje significativo a partir de la construcción propia del conocimiento por parte de los estudiantes y de la interacción con los demás factores socioculturales y ambientales de su entorno (28). 
Zeballos (25) en su estudio confirma una tendencia a reconocer en las plantas el simbolismo hacia la trascendencia, y lo fundamental según refiere Ismael (32), es que el uso adecuado expresado en concentraciones, formas de administración y métodos de extracción podrían resultar como medio paliativo para el tratamiento de diferentes patologías, sin dejar de considerar a los medicamentos como primera línea de elección

Finalmente, Sauvé (35) afirma que la educación ambiental donde incluye también a las plantas medicinales en su contexto, no es una educación temática colocada dentro de una pluralidad de otras. Se centra en una de las tres esferas (concéntricas) de interacciones que se encuentran en la base del desarrollo personal y social, en estrecha conexión con la esfera de la relación consigo mismo (zona de construcción de la identidad), y con la esfera de la relación con el otro humano (zona de desarrollo de las relaciones de alteridad), existe la esfera de la relación con el ambiente, con lo otro no humano, con el estilo de vida compartido entre lo humano y las otras formas de vida.

De manera general, la intervención educativa mejoró significativamente la cultura ambiental y fitoterapéutica de los estudiantes del nivel secundario de una institución educativa de la provincia de Julcán, La Libertad a través del incremento de las puntuaciones en las dimensiones calidad ambiental y calidad de vida en el pretest (68,2 puntos) y postest (117 puntos) del grupo control y experimental.

\section{REFERENCIAS BIBLIOGRÁFICAS}

1. Calvo S. Educación Ambiental. Conceptos y Propuestas. Madrid: C. L. S. 1994.

2. Caride J. La educación ambiental como investigación educativa. Ambientalmente sustentable: Revista científica Galego-Lusófona de Educación Ambiental. 2007; $1(3), 33-55$.

3. Orejuela E. Impacto del programa de educación ambiental (EDUCAVERDE) en las actitudes y cultura ambiental de niños de $1^{\circ}$ grado de secundaria de dos colegios piloto de la ciudad de Trujillo - Perú. Tesis para optar el grado de Doctor en Medio Ambiente. Trujillo: Universidad Nacional de Trujillo. 2007.

4. Meráz P. Cultura ecológica para el cuidado del medio ambiente en una empresa dedicada a enseres domésticos. Tesis para optar el grado de Magíster en Ciencias de la Administración. San Nicolás de los Garza: Universidad Autónoma de Nuevo León. 2003.

5. Castillo E, Martínez I. Manual de fitoterapia. Barcelona: Elsevier - Mason. [en línea] 2007; [fecha de acceso 27 de octubre del 2014]. Disponible en: http:// books.google.com.pe/books?id=SgZjLFGBAAcC\&printsec=frontcover\&dq=fitoterapia\&source=gbs_similarbooks_s\&cad=l $\# \mathrm{v}=$ onepage $\& \mathrm{q}=\& \mathrm{E}=$ false. 20 enero, 2010.

6. Castillo E. Estudio fitoquímico de Plukenetia volubilis L. y su efecto antioxidante en la lipoperoxidación inducida por Fe/ascorbato en Rattus rattus var. albinus. Tesis 
para optar el grado de maestro en Farmacia y Bioquímica. Trujillo: Universidad Nacional de Trujillo. 2008.

7. Castillo E, Castillo S, Reyes C. Efecto musculotrópico uterina del decocto de Nasturtium officinale en Rattus rattus var. albinus. Revista de Investigaciones Aplicadas Sciendo. 2009; 12(2), 14-17.

8. Castillo E, Castillo S, Reyes C. Efecto protector de Desmodium molliculum L. en la injuria aguda de mucosa gástrica inducida por etanol en Rattus rattus var. albinus. Revista de Investigaciones Aplicadas Sciendo. 2009; 12(1), 25 - 28.

9. Lozoya X, Cañigueral S. Sobre la Fitoterapia. Boletín Latinoamericano y del Caribe de Plantas Medicinales y Aromáticas 5(4), 67. [en línea] 2006; [fecha de acceso 25 de noviembre del 2013]. Disponible en: http://redalyc.uaemex.mx/redalyc/ pdf/856/85650401.pdf. 20 enero, 2010.

10. Alea A. La educación ambiental hacia el desarrollo sostenible. [en línea] 2005; [fecha de acceso 12 de junio del 2014]. Disponible en: http://www.monografias. com/trabajos25/educacion-ambiental/educacion-ambiental.

11. Febles M. Bases para la psicología ambiental en Cuba. La Habana: Universidad de la Habana - Cuba, Facultad de Psicología. 1997.

12. Leff E. Ciencias Sociales y Formación ambiental. España: Gedisa. 1996.

13. Cejas E, Viltres C, Feijoo M. La educación para el desarrollo sostenible y la formación por competencias laborales. Revista de Didáctica Ambiental. 4(6) [en línea] 2007; [fecha de acceso 03 de octubre del 2014]. Disponible en: http://www.didacticaambiental.com/revista/numero6/competencia.html

14. García J, Cano M. ¿Cómo nos puede ayudar la perspectiva constructivista a construir conocimiento en educación ambiental? Revista Iberoamericana de Educación, 41(1), 117- 131. [en línea] 2006; [fecha de acceso 19 de noviembre del 2014]. Disponible en: http://www.rieoei.org/rie4la05.htm. 19

15. González M. Informe sobre el Proyecto "La Educación Ambiental en Iberoamérica en el Nivel Medio”. Balance Provisional. En: Educación ambiental teoría y práctica. Revista Iberoamericana de Educación 11(1): 119 - 125. [en línea] 1996; [fecha de acceso 19 de enero del 2014]. Disponible en: http://www.rieoei.org/oeivirt/ riella06.htm. 19 enero, 2010.

16. Sucar S. La educación ambiental: una prioridad del programa institucional de Medio Ambiente de la Universidad de Guanajuato, México. Universidad de Guanajuato. En: Memorias del IV Congreso Iberoamericano de Educación Ambiental. La Habana, Cuba, 2 al 6 de junio de 2003. 2003. 
17. Terrón E. La educación ambiental en la educación básica, un proyecto inconcluso. Revista Latinoamericana de Estudios Educativos. 2004; 34(4): 107-164.

18. Nodarse N. La educación ambiental una vía para la participación popular. Tesis para optar el grado de Magíster en Desarrollo Social Caribeño. Habana: Universidad de la Habana. 2005.

19. Molina Y. Programa de educación ambiental para la cuenca del rio Mucujún, una ventana de extensión universitaria. Revista Venezolana de Educación Educere 2006; 10(34), $471-482$.

20. Fernández M, Fallas Y. Educación Ambiental: Cómo elaborar un programa de actividades ambientales para el centro educativo. En: Memorias del IX Congreso Nacional de Ciencias. Costa Rica, 24 y 25 de agosto. 2007.

21. Carrero A, García M. Programa educativo ambiental para las poblaciones de la Zona Costera del Estado Miranda. Revista de Investigación. 2008; 63(1): 1.

22. Boada D, Escalona J. Pedagogía ambiental en Venezuela y educación inicial. Revista Venezolana de Educación Educere. 2004; 8(25): 181 - 186.

23. Echarri F, Puig J. Educación ambiental y aprendizaje significativo. Seguridad y medio ambiente. 2008; 112(1).

24. González, M. Principales tendencias y modelos de la Educación Ambiental en el sistema escolar. En: Educación ambiental teoría y práctica. Revista Iberoamericana de Educación. 11(1), 12 - 17, 25 - 34. [en línea] 1996; [fecha de acceso 03 de octubre del 2013] Disponible en: http://www.rieoei.org/oeivirt/riella01.htm.

25. Zeballos M. Impacto de un proyecto de educación ambiental en estudiantes de un colegio en una zona marginal de Lima. Tesis para optar el grado de Magister en Gerencia Social. Lima: Pontificia Universidad Católica del Perú. 2005.

26. Gómez L. Sistematización de la implementación del programa de educación ambiental de la Reserva Nacional de Salinas y Aguada Blanca. [en línea] 2007; [fecha de acceso 19 de octubre del 2014] Disponible en: http://www.profonanpe.org. pe/gpan/documentos/Com_Educ_Amb/Sistemat_Edu_Amb_\%20RNSAB.pdf. 20 enero, 2012.

27. Beltram R. Programa de mejoramiento ambiental para la campiña de Moche (La Libertad, Perú). Tesis para optar el grado de Doctor en Medio Ambiente. Trujillo: Universidad Nacional de Trujillo. 2005.

28. Vidal L, Raimundez E. Modelo educativo ambiental para la unidad educativa Liceo San José de los Teques, estado Miranda, Venezuela. Revista Venezolana de Educación Educere 2009; 13(46), 605 - 613. 
29. Bernal A, Meléndez A. Diseño de un programa de educación ambiental para la conservación de la avifauna, dirigido a la comunidad de Barra de Potosí, Guerrero. Tesis para optar el título de Biólogo. México D.F: Universidad Autónoma Metropolitana. 2010.

30. Novo M. La educación formal y no formal: dos sistemas complementarios. En: Educación ambiental teoría y práctica. Revista Iberoamericana de Educación 11(1): 75 - 79. [en línea] 1996; [fecha de accesol9 de octubre del 2014]. Disponible en: http://www.rieoei.org/oeivirt/riella02.htm.

31. Andraca C, Sampedro M. Programa de Educación Ambiental para incidir en la actitud del manejo de Residuos Sólidos Urbanos (RSU) de estudiantes del nivel medio superior. Revista Iberoamericana de Educación 56(3). [en línea] 2011; [fecha de acceso 15 de junio del 2014] Disponible en: http://www.rieoei.org/deloslectores/4012Andraca.pdf

32. Ismael M. Fitofármacos. Intervención Educativa. Sector Vargas. Enero - julio 2007. República Bolivariana de Venezuela. [en línea] 2008; [fecha de acceso 19 de julio del 2014]. Disponible en: http://files.sld.cu/mednat/files/2012/01/fitofarmacos-intervencion-educativa.pdf

33. Mostacero J. Características edafoclimáticas y fitogeográficas de las plantas medicinales del dominio andino nor occidental del Perú durante 1976 al 2004. Tesis para optar el grado de Doctor en Medio Ambiente. Trujillo: Universidad Nacional de Trujillo. 2005.

34. Rengifo A, Flores M, Guevara F. Programa educativo sobre manejo sostenible de la biodiversidad en estudiantes del colegio agropecuario El Milagro - San Juan, Maynas, [en línea] 2008; [fecha de acceso 03 de octubre del 2013]. Disponible en: https://docs.google.com/viewer?a=v\&q=cache:695CloEfIRkJ:www.unapiquitos.edu.pe/oficinas/iiunap/archivos/2009/agronomia/ARTICULO\%2520CIENTIFICO-DRA.\%2520ANA\%2520MARIA\%2520RENGIFO.docx

35. Sauvé L. La educación ambiental y la globalización: Desafíos curriculares y pedagógicos. Revista Iberoamericana de Educación 41(1), 93 - 95. [en línea] 2006; [fecha de acceso 06 de octubre del 2014]. Disponible en: http://www.rieoei.org/ rie4la03.htm. 